\title{
América Latina en estado de misión
}

\author{
Víctor Codina, S.J., \\ Universidad Católica Boliviana, \\ Centro de Espiritualidad Ignaciana, \\ Cochabamba, Bolivia.
}

\section{De la Lumen Gentium al decreto Ad Gentes}

En una reunión del equipo pastoral que trabajamos en la parroquia suburbana de Santa Vera Cruz, ubicada en la zona del Sur, la más pobre de la ciudad de Cochabamba, en Bolivia, unas religiosas recién llegadas para vivir insertas en un barrio muy pobre, se cuestionaban sobre cómo actuar pastoralmente con la gente del barrio. Es un barrio de reciente formación, sus habitantes son emigrantes provenientes de otras zonas del país. En el barrio no hay agua, ni alcantarillado, ni electricidad. Hay problemas sobre loteamiento de las tierras y recogida de basuras. Mucha gente desocupada vive de una economía sumergida.

Con un gran sentido común, humano y cristiano, las religiosas habían optado por acercarse primero a la gente, escuchar y conocer sus problemas, ser buenas vecinas, visitar las casas, reunir a los niños, asistir a las asambleas de las juntas vecinales... Más adelante empezarían a formar comunidad, buscarían un lugar de reunión, evangelizarían a los niños.

Entonces intuí claramente, y así se lo comuniqué a los participantes de la reunión pastoral, que había que pasar de la constitución Lumen gentium al decreto Ad gentes. Sólo lo insinué, sin explicarlo en detalle, y seguramente no se entendió mi propuesta. Quisiera ahora desarrollar y justificar qué significa y por qué pasar de la Lumen Gentium al decreto Ad gentes.

\section{Iglesia, ¿qué dices de ti misma?}

Pablo VI en su discurso de apertura de la segunda sesión del Vaticano II pidió a los obispos que trabajaban el esquema sobre la Iglesia, que respondiesen a 


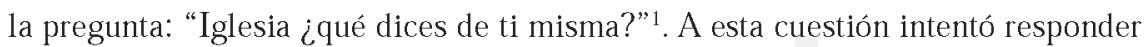
el Vaticano II con la constitución dogmática Lumen Gentium: la Iglesia radica en el misterio trinitario de Dios (LG I), es pueblo de Dios, un pueblo mesiánico que tiene a Cristo por cabeza (LG II), llamado a la santidad (LG V), que camina hacia la escatología (LG VII), constituido por jerarquía (LG III), laicos (LG IV) y vida religiosa (LG VI), y que tiene a María por modelo, prototipo y signo de esperanza (LG VIII). Esta Iglesia de Cristo que subsiste en la Iglesia católica (LG 8), tiene estrechos vínculos con los cristianos no católicos (LG 15), pero también se relaciona con los no cristianos, ya que Dios quiere que todos se salven y la divina providencia no niega sus auxilios necesarios para la salvación a cuantos se esfuerzan por vivir una vida recta (LG 16).

Lumen Gentium presenta de hecho una Iglesia ya establecida y plenamente constituida, tal como se considera que existe en los países de tradición católica, aunque reconoce el carácter misionero de la Iglesia, la necesidad de enseñar y bautizar a todas las gentes, conforme al mandato evangélico (Mt 28, 18-20), de enviar evangelizadores a todo el mundo (LG 17).

Gaudium et spes reflexionará sobre la Iglesia en el mundo contemporáneo, la Iglesia "ad extra", pero la dimensión propiamente misionera de la Iglesia se expone en el decreto Ad gentes. Este decreto en el transcurso de las discusiones, por influjo sobre todo de Congar y Ratzinger, pasó de ser un texto sobre "las misiones" a convertirse en un decreto sobre la actividad misionera de toda la Igle$\mathrm{sia}^{2}$. La Iglesia peregrina es misionera por su misma naturaleza y esta dimensión misionera nace del designio del Padre (AG 2), de las misiones del Hijo y del Espiritu (AG 3-4) y se dirige a toda la humanidad. Su finalidad es la evangelización y la implantación de la Iglesia en los grupos humanos donde todavía no ha arraigado la fe. Se refiere a los territorios llamados comúnmente "misiones", a los grupos humanos que no creen en Cristo. El decreto distingue claramente la actividad misionera en los territorios que todavía no conocen a Cristo y en los cuales todavía no hay una Iglesia local madura, de la actividad pastoral en los que ya son católicos (AG 6).

Frente a estos pueblos a donde no ha llegado todavía el anuncio del evangelio, Ad gentes propone una pedagogía misionera peculiar: testimonio de vida, diálogo cultural, reconocer las semillas de la palabra allí presentes (LG 11), solidaridad y caridad sobre todo con los pobres y afligidos como hizo Jesús en su tiempo, colaboración en lo económico y social (AG 12) y, cuando sea oportuno, anuncio del evangelio y llamado a la conversión (AG 13), admisión al catecu-

1. Pablo VI, Discurso de Apertura de la segunda sesión del Concilio Vaticano II, 29 septiembre de 1963.

2. J. Gorsky, Lo que cada católico debe saber sobre "las misiones", la actividad misionera, la misión "ad gentes" y la evangelización, Cochabamba, 2007, pp. 6-8. 
menado y a la iniciación cristiana como en la Iglesia primitiva (AG 14), formación de la comunidad cristiana (AG 15), suscitar y cultivar los ministerios para constituir un clero local ( $A G$ 16), formar catequistas laicos ( $A G$ 17), promover la vida religiosa tanto activa como contemplativa ( $A G$ 18), hasta ir formando Iglesias particulares arraigadas y establecidas que puedan a su vez ser misioneras en otros territorios por evangelizar (AG 19-22).

El resto del decreto trata de los misioneros y de los institutos que trabajan en las misiones (AG 23-27), de la ordenación de la actividad misionera (AG 28-34) y de la cooperación de toda la Iglesia con estas misiones (35-42), en estrecha conexión con la congregación para la propagación de la fe (AG 29).

Pero este decreto, aunque pasa de las "misiones" a la "actividad misionera" de toda la Iglesia, distingue claramente la actividad pastoral entre los fieles de la actividad misionera entre los infieles. El concilio Vaticano II distingue, pues, claramente dos situaciones o momentos eclesiales diferentes: la Iglesia establecida y constituida (LG) y la Iglesia que envía misioneros a territorios no evangelizados donde ella no está presente (AG). Lumen Gentium posee un carácter más estático y conservador, mientras $A d$ gentes es más dinámico, constituye una verdadera eclesiogénesis, se nos describe cómo hacer nacer la Iglesia en lugares donde no estaba presente.

Los dos documentos se refieren a dos sectores eclesiales claramente diferenciados, que corresponden básicamente a zonas geográficas determinadas: Europa y América, por una parte, que necesitan una atención pastoral ordinaria (LG), Asia y África, por otra parte, que requieren una actividad misionera (AG)

\section{La situación eclesial está cambiando}

Después de casi 50 años del Vaticano II la situación de la Iglesia ha cambiado profundamente en todo el mundo.

Esta mutación no se debe sólo al Vaticano II, como algunos han hecho creer, sino que ya venía de lejos. Ya en 1943 el libro del abbé Godin Francia ¿paîs de misión? alertó sobre el fenómeno de la descristianización de Francia, nación considerada durante siglos como la hija predilecta de la Iglesia. Muchos se escandalizaron, pero el proceso ha seguido adelante.

K. Rahner, ya desde antes del Concilio, afirmaba que se estaba pasando de una Iglesia de masas (Volkskirche), fuertemente unida a la sociedad y a la cultura cristiana, típica de la época de cristiandad, a una Iglesia de pequeña grey, minoritaria, a un cristianismo de diáspora, donde los cristianos lo serían no por simple tradición sociológica, sino por convicción personal, con una verdadera calidad cristiana ${ }^{3}$. Se hizo célebre la afirmación del gran teólogo alemán de que el cris-

3. K. Rahner, Cambio estructural en la Iglesia, Madrid, 1974, pp. 31-43. 
tiano del siglo XXI o será místico o no será cristiano4. Se pasa de una Iglesia de masas a una comunidad de creyentes. La iglesia de cristiandad, ciertamente en muchos lugares, todavía no ha desaparecido ni hay que hacerla desaparecer, pero - dice Rahner - tiene un carácter residual, por más que a algunos esto les duela y no lo acepten, añorando siglos pasados.

El teólogo Ratzinger hace ya años, en una entrevista con P. Seeweld, afirmaba en términos proféticos que la Iglesia del futuro se reduciría numéricamente, sobre todo en Europa, y que ya no se podría identificar la Iglesia con el pueblo. Le llovieron muchas críticas antes estas afirmaciones, pero la historia le ha dado la razón $n^{5}$.

La división tan neta del Vaticano II entre una Iglesia ya establecida y estructurada (LG) y una Iglesia en germen en zonas geográficas y jurídicas de misión a pueblos "paganos" (AG), ha desaparecido en buena parte. Ahora, la Iglesia se halla toda ella "en estado de misión", misión no sólo para a zonas geográficas alejadas donde viven los pueblos llamados "infieles o paganos" (lo que se llamará luego misión "ad gentes"), sino misión hacia grupos humanos muy diversos que viven en territorios de tradición católica pero que están alejados de la Iglesia. Es lo que P. Suess llama misión "inter gentes".

En efecto, muchos territorios tradicionalmente cristianos-católicos son ahora tierra de misión: crece el número de católicos no practicantes, la fe ya no se transmite de padres a hijos, muchos abandonan la Iglesia en un cisma silencioso, otros apostatan públicamente, disminuyen aceleradamente las vocaciones a la vida religiosa y al ministerio ordenado, aumentan los cristianos "sin Iglesia", hay crisis de las instituciones religiosas, hay crisis de fe en Cristo y en Dios, crece el indiferentismo y el agnosticismo.

La Lumen gentium, al hablar del Pueblo de Dios, reconocía diversas formas de participación de los cristianos en la Iglesia de Cristo (LG 13), pero no contemplaba estas diversas formas de pertenencia eclesial entre los mismos católicos. La realidad eclesial se ha complejizado y problematizado.

Pablo VI ya habló de pertenencia "parcial" a la Iglesia y de "cristianos no practicantes" (EN 21). Los sociólogos de la religión establecen diversas tipologías según la praxis eclesial: militantes, practicantes habituales, practicantes ocasionales y disidentes (Le Bras); el practicante moderno es como un peregrino, voluntario, autónomo, variante, individual, móvil y excepcional (D. HervieuLéger); aumenta la creencia sin pertenencia (G. Davie). Los teólogos también estudian esta situación y hablan de "Iglesia abierta" (K.Rahner), "Iglesia del

4. K. Rahner, Escritos de teologia, VII, Madrid, 1967, p. 25.

5. P. Seeweld, Dios y el mundo, cita en Quaderns de pastoral, Barcelona, $\mathrm{n}^{0}$ 206-207 (2007) 72 . 
umbral" (église du seuil) o Iglesia "catecumenal" (Y. M. Congar), de pertenencia débil (S. Dianich), de cristianos de las cuatros estaciones de la religiosidad popular (D. Borobio) ${ }^{6}$.

Juan Pablo II, en su exhortación post-sinodal Chrisfideles laici de 1989, después del sínodo de los laicos, escribe unos párrafos de gran lucidez eclesial y pastoral:

Enteros países y naciones, en los que en un tiempo la religión y la fe cristiana fueron florecientes y capaces de dar origen a comunidades de fe viva y operativa, están ahora sometidos a dura prueba e incluso alguna que otra vez son radicalmente transformados por el continuo difundirse del indiferentismo, del secularismo y del ateísmo. Se trata en concreto del Primer mundo, en el que el bienestar económico y el consumismo - si bien entremezclado con espantosas situaciones de pobreza y miseria - inspiran y sostienen una existencia vivida "como si no hubiera Dios" (ChL 34).

Este proceso de descristianización desde 1989 a nuestros días sin duda se ha agravado y difundido mucho más todavía.

Juan Pablo II contrapone después a esta situación de descristianización, típica del primer mundo, la de otros países donde la tradición cristiana está viva, aunque amenazada:

En cambio, en otras regiones o naciones todavía se conservan muy vivas las tradiciones de piedad y de religiosidad popular cristianas; pero este patrimonio moral y espiritual corre hoy el riesgo de ser desperdigado bajo el impacto de múltiples procesos, entre los que destacan la secularización y la difusión de las sectas. Sólo una nueva evangelización puede asegurar el crecimiento de una fe límpida y profunda, capaz de hacer de estas tradiciones una fuerza de auténtica libertad" (Ch L 34).

Junto a esta problematización de los cristianos que tradicionalmente formaban parte de la Iglesia, también la misión "ad gentes" ha sufrido un cambio. A1 abandonar el Vaticano II el viejo axioma "extra ecclesiam nulla salus" y reformularlo positivamente, afirmando que la Iglesia es "sacramento universal de salvación" (LG 1; 9; 48) y que el Espíritu Santo ofrece a todos la posibilidad de salvación asociándolos al misterio pascual de una forma de sólo Dios conocida (GS 22), en muchos sectores cristianos el tema misional ha entrado en crisis. Se ha pasado del "siglo de las misiones a la era de las religiones" (J. Masiá), y se abre una problemática totalmente nueva frente a la misionología clásica: diálogo intercultural e inter-religioso, visión positiva de las religiones no cristianas, libertad religiosa, el problema de la única mediación de Cristo, la posibilidad de

6. Sobre esta cuestión puede consultarse S.Pié-Ninot, Eclesiología, Salamanca, 2007, pp. 281-286. 
considerar como inspiradas las escrituras religiosas de las religiones de la humanidad... Rahner, comentando que la Iglesia es sacramento de la salvación para el mundo, reconoce que la mayoría de la humanidad se salva fuera de la institución eclesial ${ }^{7}$. Ahora parece que el problema fundamental ya no son tanto los que están lejos (los paganos, "ad gentes"), pues se pueden salvar fuera de la Iglesia, sino los alejados de dentro de la misma Iglesia.

Esto explica que en 1990, Juan Pablo II en su encíclica Redemptoris missio, a los 50 años del Decreto conciliar $A d$ gentes, quisiera relanzar la misión "ad gentes", que había sido un tanto cuestionada en los últimos años. En dicho documento establece una triple tipología pastoral de la Iglesia de hoy:

a) actividad pastoral en países que tienen comunidades cristianas formadas sólidamente,

b) nueva evangelización para países de antigua cristiandad o Iglesias jóvenes que han perdido el sentido de la fe y las exigencias del evangelio,

c) la misión "ad gentes", en los países donde Cristo y el evangelio no son conocidos (RMi 33).

Estamos, pues, viviendo un proceso de cambio: del esquema eclesiológico claro del Vaticano II que establecía una neta división entre la Iglesia ya establecida de los países de tradición católica, donde se requiere una pastoral tradicional de conservación (LG) y la Iglesia que se tenía que implantar en tierras de misión (AG). Estamos viviendo una situación mucho más compleja y problematizada, con otros elementos nuevos. No sólo hay una actividad pastoral para la Iglesia ya establecida y una actividad misional para los no cristianos, sino que se necesita una nueva evangelización para territorios que han perdido el sentido de la fe o las exigencias del evangelio. En este sentido afirma Benedicto XVI:

El campo de la misión "ad gentes" se ha ampliado notablemente y no se puede definir sólo basándose en consideraciones geográficas o jurídicas. En efecto, los verdaderos destinatarios de la actividad misionera del pueblo de Dios no son sólo los pueblos no cristianos y las tierras lejanas sino también los ámbitos socioculturales y, sobre todo, los corazones ${ }^{8}$.

\section{En busca de las causas de esta nueva situación}

Las causas de esta nueva situación de crisis eclesial son muy complejas y de distinta índole. Nos limitaremos tan sólo a insinuar algunos aspectos? ${ }^{9}$.

7. K.Rahner, op. cit., p. 78

8. Benedicto XVI, Discurso a los miembros del Consejo Superior de Obras Misionales Pontificias, 5 de mayo de 2007, citado en el Documento de Aparecida 374.

9. Puede verse para este tema V. Codina, Sentirse Iglesia en el invierno eclesial, Cristianisme i justicia, Barcelona, 2006. 
Para muchos sectores eclesiales, sobre todo del primer mundo, la desafección eclesial es debida a los recientes escándalos sexuales causados por algunos ministros de la Iglesia, a la falta de respeto a los derechos humanos dentro de la misma institución eclesial, a las posturas conservadoras y rígidas del magisterio eclesiástico en materias de moral sexual, familiar y bioética, a la poca apertura de la Iglesia a nuevas formas de ministerio ordenado, a la discriminación de la mujer en la Iglesia, al autoritarismo y centralismo romano, al poco respeto a las Iglesias locales, etc

Pero hay otros problemas eclesiales y teológicos más de fondo, que van más allá de las cuestiones de sexualidad, gobierno o derechos humanos.

En el Vaticano II oficialmente la Iglesia se aleja del paradigma de cristiandad vigente desde Constantino hasta el siglo XX. Pero el nuevo paradigma de Iglesia pueblo de Dios todavía no ha sido plenamente recibido en muchos sectores $y$ todavía no se aceptan las consecuencias que este cambio va a suponer. E1 paso de una Iglesia clerical, donde los laicos son sujetos pasivos, a una Iglesia laical donde todos los bautizados se sienten responsables de la comunidad eclesial, todavía es un proceso lento, y además esta toma de conciencia de la Iglesia del laicado va a conllevar una disminución de vocaciones al ministerio sacerdotal y sobre todo a la vida religiosa.

Formar parte del ministerio ordenado tenía en la época de cristiandad un gran apoyo social, era algo socialmente reconocido y estimado. Ahora es cuestionado y minusvalorado. De hecho, las vocaciones sacerdotales han disminuido drásticamente en los países de vieja tradición católica, muchas parroquias han quedado sin sacerdote.

La vida religiosa también ha quedado afectada. La vida religiosa nació precisamente como respuesta profética al surgimiento de la Iglesia de cristiandad (siglo IV), y los sucesivos ciclos de vida religiosa (siglos XII, XVI, XVIII, XIX) querían responder a situaciones críticas de la Iglesia de cristiandad, a las que ni la Iglesia jerárquica, ni los laicos, ni los estados eran capaces de responder. Una vez superada esta etapa eclesial de cristiandad, la vida religiosa sufre en los países del primer mundo una disminución numérica de efectivos, mientras que las vocaciones religiosas se mantienen y aumentan en lugares donde todavía está vigente la Iglesia de cristiandad.

La gran masa de cristianos bautizados ya no podrá apoyarse en el respaldo sociológico y cultural de una "sociedad cristiana", como sucedía en la época de cristiandad, sino que tendrá que optar personalmente en una sociedad con un gran abanico de ofertas religiosas y culturales. Lo que mantenía la unidad católica en la Iglesia de cristiandad no era necesariamente la fe sino una cierta homogeneidad cultural, que ahora se ha roto ${ }^{10}$.

10. K.Rahner, op. cit., p. 32 
Además, si hay salvación fuera de la Iglesia, ¿qué sentido y función tiene la Iglesia? Esto está ligado al tema de la cristología que se ha convertido hoy en el ojo del huracán, no sólo por los nuevos avances exegéticos, sino por la problemática surgida ante el pluralismo religioso, como ya hemos insinuado antes. De cara a la vida eclesial es cuestionante el silencio que durante siglos ha reinado sobre el concepto de reino de Dios, que fue el centro de la predicación y anuncio de Jesús, pero luego fue desapareciendo o transformándose. El reino de Dios se identificó con Jesús, luego con la Iglesia (sobre todo con su estructura jerárquica), luego con la escatología. El redescubrimiento del reino obliga a resituar la eclesiología de forma diferente a la habitual ${ }^{11}$. Lo mismo cabe decir acerca del redescubrimiento de la pneumatología.

Pero todavía no hemos tocado fondo. La crisis ya no es de Iglesia, ni de Cristo, sino de Dios. Las huellas de Dios están muy ocultas en un mundo secularizado. Por esto no es casual que Pablo VI, que en el Vaticano II pedía que la Iglesia dijese qué pensaba de sí misma, después del Concilio, en una Semana Social de Francia pidiese que la Iglesia dijese qué pensaba de Dios. Y Juan Pablo II exhorta a los teólogos que abran caminos de acceso al misterio de Dios ${ }^{12}$.

Esta crisis de fe, silencio y eclipse de Dios, esta noche oscura, parece responder a una crisis epocal que según algunos (K. Jaspers) supone la crisis de un período axial de creencia en Dios que ha estado vigente durante los últimos 6000 años. Otros hablan de una espiritualidad laica, sin creencias, sin religiones, sin dioses, ya que hemos superado la era pre-industrial (M. Corbí). La modernidad ilustrada, secular y globalizada ha producido una gran conmoción, un auténtico "tsunami" (J. Comblin) a la Iglesia tradicional pre-moderna de cristiandad. Ahora la trascendencia de Dios queda cuestionada, la religión parece reducirse a la inmanencia, a una autonomía encerrada en sí misma (J. Martín Velasco). Por eso aumenta el agnosticismo, el nihilismo, la indiferencia. Ni siquiera los grandes ateismos del siglo XIX interesan. Se requiere un nuevo paradigma religioso.

En este contexto se explica la afirmación tantas veces citada de K. Rahner de que el cristianos del siglo XXI o será místico o no será cristiano ${ }^{13}$. El mismo Rahner se pregunta si hay que buscar salvar a los perdidos o conseguir testigos que sean signos de la gracia de Dios que actúa en todo el mundo ${ }^{14}$.

\section{Problemática eclesial y pastoral de América Latina}

A todo lo dicho hasta ahora sobre el malestar eclesial se podría objetar que esta situación refleja más al primer mundo que a América Latina, que mantiene

11. J. Sobrino, La fe en Jesucristo, Madrid, 1999, pp. 469-470

12. Juan Pablo II, Discurso a los teölogos en Alttöting, noviembre de 1980.

13. Cfr nota 4.

14. K.Rahner, op. cit., p. 78. 
un profundo sentido religioso, está mucho menos secularizada que las naciones técnicamente avanzadas, no vive tan fuertemente el proceso de descristianización que se da por ejemplo en Europa occidental. América Latina era considerada hasta hace poco como una especie de "Amazonia católica", un pulmón oxigenante de la Iglesia católica frente a la asfixia religiosa del primer mundo. Esto hoy es muy discutible.

América latina forma parte de estas regiones o naciones que, según Juan Pablo II, conservan vivas las tradiciones de la piedad popular. Pero a pesar de ello hay que reconocer que América Latina y el Caribe es una de estas Iglesias jóvenes que han perdido el sentido de la fe y la exigencia del evangelio. Por esto el magisterio latinoamericano desde hace tiempo habla de la necesidad de una nueva evangelización.

Ya Medellín en 1968 habló de la necesidad de una evangelización, re-evangelización y nueva evangelización para América Latina (Medellín 6, 8; Mensaje a los pueblos de América Latina).

Juan Pablo II en Haití en 1983 lanzó el programa de una nueva evangelización para América Latina para prepararse a los 500 años de la primera evangelización y dijo que la nueva evangelización debía ser nueva en el ardor, en sus métodos y en sus expresiones ${ }^{15}$.

La nueva evangelización fue el tema central de Santo Domingo, que un sector la entendió como diferente de la propugnada por Medellín, a la que consideraban excesivamente horizontalista y sociológica; por esto en Santo Domingo se cambió la metodología típica latinoamericana del ver, juzgar y actuar.

\section{Perspectiva misionera desde Aparecida}

Aparecida, en 2007, vuelve a insistir en el tema de "Discípulos y misioneros de Jesucristo para que nuestros pueblos en Él tengan vida”. El tema de la misión es central en Aparecida. A su luz podemos establecer las siguientes afirmaciones.

1. La fe y la religiosidad popular son la gran riqueza de América Latina

Tanto el discurso del papa Benedicto XVI como el documento conclusivo de Aparecida ponderan la riqueza y valor de la religiosidad popular:

- es el precioso tesoro de la Iglesia católica de América Latina, refleja la sed de Dios que sólo los pobres y sencillos reconocen (Aparecida 258; cfr. 549).

- esta religiosidad popular confiesa amor a Cristo sufriente, profesa su fe en el Dios de la compasión, del perdón y la reconciliación, en el Señor presente en la eucaristía, en el Dios cercano a los pobres, tiene una profunda devoción

15. AAS 75 (1983) 778 . 
a la Virgen en sus diversas advocaciones (Aparecida 7, con cita del discurso inaugural 1).

- esta fe está presente en la lucha por la justicia, la esperanza contra toda esperanza, la alegría de vivir aun en condiciones muy difíciles, se manifiesta en el arte, el lenguaje, tradiciones y fiestas (Aparecida 7), se expresa en las fiestas patronales, novenas, rosarios, vía crucis, procesiones, danzas religiosas, cariño a santos y ángeles, promesas, oraciones en familia, peregrinaciones a santuarios (Aparecida 259), donde se realizan historias de conversión y perdón (Aparecida 260).

- no es una espiritualidad de masas, sino que cada persona la vive de forma personal en su vida cotidiana, con gestos muy concretos hacia el Señor o María (Aparecida 261).

- es punto de partida para que la fe del pueblo madure, para que se profundice su riqueza evangélica (Aparecida 262), consiguientemente no se puede devaluar como un modo secundario de vivir la fe cristiana, pues en ella hay una experiencia teologal, una sabiduría sobrenatural, una verdadera espiritualidad encarnada en la cultura de los sencillos (Aparecida 263), un modo legítimo de vivir la fe, una síntesis entre culturas y fe cristiana (Aparecida 264).

en ella se expresa el alma de los pueblos latinoamericanos (Aparecida 258), hace tomar consciencia de la común condición de hijos de Dios y de la común dignidad, más allá de las diferencias sociales, étnicas o de otro tipo (Aparecida 37; cfr 99b).

\section{La fe de América Latina se debilita y erosiona}

Sin embargo, Benedicto XVI, tras haber alabado la rica tradición de la fe popular cristiana de América Latina, afirma: "Se percibe, sin embargo, un cierto debilitamiento de la vida cristiana en el conjunto de la sociedad y de la propia pertenencia a la Iglesia católica" (discurso inaugural, 2). Y el documento conclusivo, a pesar de reconocer las realizaciones positivas de la Iglesia en sus diversas pastorales (Aparecida 98-99), constata una crisis y debilitamiento de la fe cristiana:

- la fe se erosiona (Aparecida 13; 38).

existe mucho individualismo, débil pertenencia a la Iglesia, sacramentalismo, poco compromiso de los laicos, disminución de vocaciones ministeriales, marginación de la mujer, clericalismo, materialismo, falta de sentido de la trascendencia, abandono de prácticas religiosas, paso a otros grupos religiosos, agnosticismo (Aparecida 100).

- llama la atención la multitud de bautizados no suficientemente evangelizados (Aparecida 293). 
- el continente con mayor número de católicos es el de mayor desigualdad social (Aparecida 527).

- se advierte que una fe reducida a prácticas de devoción fragmentadas, participación ocasional en algunos sacramentos, repetición de principios doctrina les y moralismo, no resistirá a la larga el embate del tiempo (Aparecida 12).

Así como K. Rahner afirmaba que la mayoría de la humanidad se salva al margen de la pertenencia a la Iglesia, podemos añadir nosotros, que desde América Latina, la mayoría de los cristianos católicos viven su fe y se salvan no fuera, pero sî al margen de la Iglesia institucional, es decir, viven su fe cristiana en el umbral de la Iglesia oficial, forman parte de "la Iglesia informal", poseen una identificación parcial con la Iglesia, viven mayormente en el mundo de la religiosidad popular: los sacramentales y los cuatro sacramentos de las estaciones de la vida ${ }^{16}$.

Sería largo examinar las causas de este deterioro de la fe en América Latina. Además de las causas generales y universales, tanto socio-culturales como teológico-eclesiales, que hemos enumerado antes al hablar de la Iglesia universal, habría que añadir las peculiares de América latina.

Hay sin duda causas históricas ligadas a la ambigüedad de la primera evangelización, donde se mezclaron luces y sombras (Puebla 13). Pero hay que reconocer causas más recientes, como la débil implantación de la eclesiología y de la pastoral del Vaticano II en América Latina, y sobre todo la critica sistemática que ha sufrido el intento de una Iglesia liberadora, tanto de parte de sectores políticos y económicos del continente y del mundo, como de parte de sectores de la Iglesia romana y latinoamericana. Hoy padecemos las consecuencias de todo ello.

\section{América Latina está en estado de misión}

Como consecuencia de lo anterior, todo el continente debe colocarse "en estado de misión" (Aparecida 213). De fieles bautizados hay que pasar a discípulos y misioneros.

Podemos afirmar desde esta nueva perspectiva, que también América Latina ha de pasar de la pastoral tradicional de Lumen Gentium a la misionera del decreto Ad gentes. Este decreto ha pasado de ser un documento un tanto territorial y geográfico para algunos países de misión a ser un documento emblemático, inspirador de la nueva situación eclesial: una Iglesia toda ella misionera que evangeliza nuevas situaciones de misión, los nuevos areópagos. Paulo Suess lamenta que Aparecida, que habla de esta situación misionera y cuyo tema central es la misión, no cite más que dos veces el decreto Ad gentes (Aparecida 347; 376), que podría ser la espinal dorsal de Aparecida, de una Iglesia misionera ${ }^{17}$.

16. V. Codina, "La Iglesia informal", Enfoque 139 (2007) 12-17.

17. P. Suess, Dicionario de Aparecida, Sāo Paulo, 2007, p. 25 
La religiosidad popular, la religión de los pobres, de la "Iglesia informal" que ha sobrevivido durante siglos y ha demostrado tener una gran capacidad de resistencia, ¿se podrá mantener en el futuro, resistirá al embate del tiempo, o es algo residual de la época de cristiandad que va a desaparecer? ¿Llegará a América Latina el proceso de secularización y la situación de descristianización de Europa? He aquí unas preguntas difíciles de responder pero que no nos exhiben de adoptar una postura misionera.

\section{Hay que pasar de una pastoral de conservación a una pastoral misionera}

Aparecida propone pasar de una pastoral de conservación a una pastoral misionera (Aparecida 370), estamos en estado de misión, viviendo una nueva época, un nuevo paradigma. Una pastoral misionera supone una nueva evangelización, no simplemente re-evangelizar, pues no se parte de cero (Santo Domingo 24).

Evangelizar es hacer lo que hizo Jesús con sus palabras y obras, anunciar y hacer presente el reino de Dios (Santo Domingo 279), un reino de vida (Aparecida 359,361 ), que implica la liberación de toda muerte y esclavitud, para que nuestros pueblos tengan vida. La evangelización ha de ser integral, no ha de buscar el aumento de los católicos sino el servicio al mundo, una evangelización liberadora, que incluya la opción preferencial por los pobres, la promoción humana integral y la auténtica liberación cristiana (Aparecida 146; cfr. caps. 7-8), para transformar los criterios, los valores, los intereses, los modelos de vida de la humanidad que están en contradicción con la palabra de Dios y el designio de salvación (EN 19; Aparecida 331).

Esta evangelización supone el anuncio del kerigma (EN 22), de modo que se pueda llegar a una experiencia personal con el Señor, sin la cual no es posible llegar a ser cristianos (Aparecida 243, con cita de Dios es amor, 1).

Esta experiencia espiritual ha de profundizarse en una verdadera formación cristiana en un proceso que vaya conduciendo a una conversión, al discipulado, a la inserción comunitaria y a la misión (Aparecida 278).

Esta misión se orienta al reino de Dios y a la promoción humana (Aparecida capítulo 8, 380-430), superando cualquier visión individualista, intimista y espiritualista de la vida cristiana.

Esto implica una conversión de las estructuras eclesiales actualmente existentes que ya no responden a la realidad latinoamericana de hoy.

\section{Los pobres han de tener prioridad en el proceso evangelizador}

Si la opción por los pobres forma parte de las prioridades de la Iglesia latinoamericana y del Caribe (Aparecida 391-398) y se reafirma que ellos poseen un potencial evangelizador (Aparecida 398), hay que deducir que, en este proyecto misionero, los pobres también han de tener prioridad. Este punto, que Aparecida 
no desarrolla, es muy importante, pues según como se entienda la misión evangelizadora, los pobres pueden quedar excluidos de ella.

Hay el riesgo de optar por evangelizar prioritariamente a los ricos, a los católicos de siempre, a los practicantes dominicales, que tienen mayor formación humana y cristiana (muchos de ellos han sido alumnos de nuestros colegios y universidades católicas), poseen tiempo y recursos para su formación cristiana y para colaborar apostólicamente (ser catequistas, dar cursillos...). Sin embargo, estos sectores que tradicionalmente han sido muy atendidos por la Iglesia no han logrado transformar las estructuras sociales, ni han sido agentes de cambio en la sociedad. Los cambios siempre han venido de abajo. A estos sectores no se les podrá abandonar, pero habrá que evangelizarlos desde los pobres, desde abajo, pidiendo que colaboren con los cambios que desde la base se propicien y que, por lo menos, no los frenen...

Evangelizar a los pobres tiene muchas dificultades. ¿Cómo evangelizar y formar a los pobres, que son la mayoría de América Latina y el Caribe, que constituyen la base de la "Iglesia informal", son los practicantes ocasionales de las cuatros estaciones de la vida, viven una religiosidad popular tradicional de fiestas y peregrinaciones, muchas veces conservadora, que no logra transformar sus vidas ni menos aún las estructuras sociales? Estos pobres tienen poca formación cristiana, poco tiempo para dedicarse a participar en cursos y reuniones, llegan a sus casas por las noches cansados del duro trabajo del día, dedican los fines de semana a comprar en el mercado, lavar su ropa, arreglar su casa, asistir a reuniones de barrio, hacer trabajos comunes...

Y sin embargo, estos pobres son los predilectos de Jesús, uno de cuyos signos mesiánicos es el que los pobres sean evangelizados (Lc 7, 22), a ellos han sido revelados los misterios del reino (Lc 10, 21).

La solución parece debería ser no anular su religiosidad popular típica de Cristiandad y residual, sino partir de ella para evangelizar a los pobres. Evangelizar a los pobres no equivale a "enseñarles el catecismo", sino que consiste en anunciarles que el reino de Dios que Jesús proclama es la alianza de Dios con los pobres en contra de la pobreza. Habría que evangelizar su misma religiosidad popular, iluminarla con la palabra, partiendo de sus mismas expresiones populares, anunciar el kerigma pascual, iniciarles en una experiencia espiritual de fe que lleve a la conversión personal, invitarles a formar comunidades tipo comunidades eclesiales de base. Hay que aplicar a esta pastoral misionera el itinerario misionero que propone Ad gentes (11-22).

¿No podrían servir de modelo los grupos evangélicos, pentecostales y carismáticos que han logrado interesar al pueblo pobre con la palabra, han formado comunidades vivas de culto y participación, donde los laicos se sienten protagonistas y responsables? ¿No podrían ser inspiradores para esta pastoral misionera los ejemplos 
de las misiones de franciscanos, dominicos, mercedarios, agustinos, jesuitas, del tiempo de la primera evangelización de América Latina que lograron con sus métodos de reducciones formar comunidades humanas y cristianas vivas e inculturadas, auténticas síntesis de evangelización y promoción humana? (Puebla 9).

Seguramente no todos van a entrar en este proceso, habrá que renunciar a una Iglesia de masas en América Latina (P. Trigo), pero sí surgirá una Iglesia de los pobres, sacramento de salvación y de liberación histórica, donde se muestren los valores realmente evangélicos del reino, signo de la acción del Espíritu que actúa en todo el mundo, fermento para todo el pueblo y toda la Iglesia. Vale también para nosotros lo que K. Rahner escribió hace años:

Un cristianismo vivo y concreto no puede transmitirse hoy, ni sobre todo mañana, simplemente con el poder de una sociedad cristiana homogénea, que cada vez se da en menor medida, ni con medidas administrativas de arriba, ni con la enseñanza religiosa, por mucho que se den en todas las escuelas públicas y llegue a todos los niños, sino que ha de ser llevado al futuro mediante el testimonio y la vida de una comunidad cristiana auténtica, que vive en lo concreto lo que quiere decir propiamente cristianismo ${ }^{18}$.

\section{Conclusión}

Un continente en estado de misión no puede seguir como hasta ahora con una pastoral de conservación. Esta pastoral misionera, más inspirada en Ad gentes que en Lumen Gentium, implica profundos cambios personales y estructurales en la Iglesia. Hemos de abandonar el estilo y ambiciones de la Iglesia masiva de cristiandad y volver a ser fermento para una nueva eclesiogénesis misionera desde abajo. Hemos de pasar:

de una pastoral clerical a una pastoral laical, donde los laicos, no sólo varones, sino las mujeres, asuman su responsabilidad.

- de una pastoral centrada en el templo a una pastoral centrada en las casas del pueblo.

de una pastoral eminentemente y a veces exclusivamente sacramental a una pastoral centrada ante todo en el anuncio de la Palabra y la evangelización.

de una pastoral dirigida preferentemente a los sectores de la clase media y alta a una pastoral dirigida preferentemente a los pobres.

- de una pastoral centrada en lo doctrinal y moral a una pastoral mistagógica que ante todo busca la iniciación a la experiencia espiritual y a la conversión interior.

18. K. Rahner, op. cit., p. 144. 
- de una pastoral que se limita a acoger a los que vienen a una pastoral que sale a la calle y va a buscar al pueblo, visita sus casas.

- de una pastoral centrada en la palabra oral a una pastoral que tiene en cuenta otros medios de expresión, tradicionales y modernos.

-de una pastoral individualista que no forma comunidades a una pastoral orientada sobre todo a formar comunidades.

- de una pastoral desligada de la vida, abstracta y ahistórica, que no transforma la realidad, a una pastoral que parta de la realidad, la ilumine con la palabra y conduzca a la praxis histórica, que transforme la vida de las personas y de la sociedad.

- de una pastoral centrada en la parroquia, centro de culto, a una pastoral que busque formar comunidad de comunidades.

- de una pastoral que tiene como eje básico el bautismo de los niños a una pastoral que promueva una auténtica iniciación cristiana asumida personal y libremente.

de una pastoral que añora la confesionalidad y apoyo del Estado a una pas toral para una sociedad pluralista en lo cultural y en lo religioso.

- de una pastoral romanizada y centralizada desde arriba a una pastoral que, siempre en comunión con Roma, respete la autonomía de las Iglesias locales y que sea realmente intercultural.

- de una pastoral que tiene como modelo subyacente la gran Iglesia de cristiandad medieval a una pastoral que se acerca más al modelo sociológico de los pequeños grupos, de las "sectas".

- de una pastoral basada en la pedagogía de Iglesia establecida (tipo LG) a una pastoral de pedagogía misionera en la línea del decreto Ad gentes: una verdadera eclesiogénesis.

Esto supone pasar de un continente de bautizados a un continente de discípulos y misioneros.

$* * *$

Esto es lo que quise insinuar en aquella reunión parroquial del barrio sur de Cochabamba, al decir a las religiosas que hoy había que pasar de la Lumen Gentium al Ad gentes, pero que seguramente no pudo ser comprendido. 